\title{
A Qualitative Analysis of Patient-Identified Adaptive Behaviour Changes Following Interdisciplinary Acceptance and Commitment Therapy for Chronic Pain
}

Article DOI: http://dx.doi.org/10.1002/ejp.1184

NOTICE: this is the author's version of a work that was accepted for publication in the European Journal of Pain.

Changes resulting from the publishing process, such as peer review, editing, corrections, structural formatting, and other quality control mechanisms may not be reflected in this document.

Changes may have been made to this work since it was submitted for publication. A definitive version was subsequently published and can be found at:

doi: 10.1002/ejp.1184 
RUNNING HEAD: PATIENT-IDENTIFIED ADAPTIVE BEHAVIOUR CHANGE

A Qualitative Analysis of Patient-Identified Adaptive Behaviour Changes Following Interdisciplinary Acceptance and Commitment Therapy for Chronic Pain

M. Thompson', K. E. Vowles ${ }^{2,3}$, G. Sowden ${ }^{3}$, J. Ashworth ${ }^{3}$, \& J. Levell ${ }^{3}$

${ }^{1}$ Faculty of Health and Applied Sciences, University of the West of England

${ }^{2}$ Department of Psychology, University of New Mexico

${ }^{3}$ IMPACT Pain Service, Staffordshire and Stoke-on-Trent NHS Partnership Trust,

CORRESPONDENCE AUTHOR:

Miles Thompson, PhD, DClinPsy.

CATEGORY: Original article.

FUNDING SOURCES: None.

CONFLICTS OF INTEREST: None.

STATEMENT OF SIGNIFICANCE: This study provides new qualitative insights into the patient's perspective of adaptive behavioural changes gained as part of interdisciplinary pain rehabilitation. This and future work may help provide a more detailed understanding of the processes and behaviours that result in successful rehabilitation outcomes. 


\begin{abstract}
Background: Interdisciplinary treatment programs for chronic pain have strong evidence of treatment effect both immediately after treatment and at follow up. However despite strong outcome evidence, it is less clear which specific changes in behaviour are most relevant to patients or to outcomes. Indeed, it is not unknown for clinicians and patients to have different views with regard to goals of treatment. This study sought to evaluate the patients' perspective regarding important behavioural changes that occurred while they were enrolled in a four week interdisciplinary program of Acceptance and Commitment Therapy (ACT) for chronic pain.
\end{abstract}

Methods: Qualitative data were collected during a treatment session towards the end of treatment. In total, 104 completers from 16 consecutive treatment groups contributed to a dataset consisting of 315 unique qualitative comments.

Results: Thematic analysis resulted in a theme hierarchy including: over-arching themes, mid-level themes, and sub-themes. Three overarching themes were identified: 1 . Interacting with self describing an interplay between various aspects of the individual; 2. Activity - concerning how individuals practically and sustainably undertook activities and 3. Interacting with others - exploring relationships with other people. The results section further describes the mid-level and sub-themes that cluster under the overarching themes.

Conclusions: These data provide initial insights into the patient's perspective of adaptive behavioural changes gained as part of an interdisciplinary program of chronic pain rehabilitation. Overall the data suggests the importance of a mix of both ACT-specific and more universal coping / pain rehabilitation elements. Future research may examine how these processes relate more directly to treatment outcome. 


\section{Introduction}

Interdisciplinary treatment programs for chronic pain have strong evidence of treatment effect (Fordyce, 1976; Gatchel, McGeary, McGeary, \& Lippe, 2014; Gatchel \& Okifuji, 2006; Main, Keefe, Jensen, Vlaeyen, \& Vowles, 2015; Turk \& Burwinkle, 2005). These interdisciplinary programs typically include a mix of professional disciplines (e.g., psychology, physical and occupational therapy, physicians) and offer an intensive and time-limited dose of treatment, typically several hours per day, often on multiple days each week for several weeks (British Pain Society, 2013; Gatchel, et al., 2014). Efficacy and effectiveness evidence suggests treatment completers reliably experience significant reductions in distress and disability, successful return to work, and renewed engagement in meaningful activities of daily living (Flor, Fydrich, \& Turk, 1992; Gatchel et al., 2014; Gatchel \& Okifuji, 2006; Kamper et al., 2014; Kamper et al., 2015; Turk, 2002; Turk \& Burwinkle, 2005). Long-term data further indicate good maintenance of treatment gains through follow-ups of three to seven years (Bergström, Bergström, Hagberg, Bodin, \& Jensen, 2010; Hatten, Gatchel, Polatin, \& Stowell, 2006; Jensen, Bergström, Ljungquist, \& Bodin, 2005; Vowles, McCracken, \& O’Brien, 2011).

While the outcome evidence in support of interdisciplinary rehabilitation for chronic pain is robust, it is not yet clear which specific changes in behaviour are most relevant to that outcome. In other words, while outcome evidence is strong, the process evidence suggesting how these outcomes are achieved is weaker. Similar messages can be found in the 2012 Cochrane review of evidence for adults with pain and disability, which called for less general randomised controlled trials and instead for different types of studies to help understand what components work, for which patient, on which outcome and why (Williams, Eccleston, \& Morley, 2012). There are numerous ways of pursuing a more process-orientated agenda, one is to try and assess the perspective of patients themselves. After all, it is not uncommon for clinicians and patients to have different views with regard to the goals of treatment (O'Brien et al., 2010; Thorne \& Morley, 2009). Thus, it may be useful to assess and evaluate important behavioural changes from the patient perspective. It may be that mixed method analytic approaches, which include both qualitative and quantitative evaluation of patient data, may allow for more informed and nuanced insights into the clinical 
material that patients find most compelling or useful. These methods may, in time, allow researchers to form a more informed understanding of the specific processes and behaviours to target for more successful outcomes within chronic pain rehabilitation programs.

The present analyses sought to perform such an analysis using data collected during a treatment session that took place at the end of an eight-day interdisciplinary program of rehabilitation for chronic pain. The purpose of this session was to collect and share patient perspectives on adaptive behavioural changes that they felt had been made over the course of treatment. The data from patient responses allowed for a qualitative analysis aided by descriptive statistics to help elucidate the patient-identified adaptive behaviour changes that had taken place over the course of treatment.

\section{Method}

\section{Participants}

Participants included 104 completers (65.4\% female) of an interdisciplinary program of chronic pain rehabilitation located in the Midlands of the United Kingdom. Average age was 48.0 years ( $S D$ $=11.8)$ and the sample was predominately White European in ethnicity (96.1\%). Most patients were married or co-habitating (64.4\%; Single: 19.3\%; Divorced: 11.5\%; Widowed: $4.8 \%$ ) and unemployed at the time of treatment onset $(65.0 \%$ not working; $21.2 \%$ working full or part time; $3.8 \%$ working as a "homemaker"; $11.5 \%$ no employment status identified). Modal pain duration was 6.7 years (range: $0.5-27.6$ years). The most frequently identified primary site of pain was low back: $49.0 \%$, followed by full body: $15.4 \%$, neck: $11.5 \%$, lower limb: $7.6 \%$, shoulder/upper limb: 5.7\%, mid-back: 4.9\%, abdominal: $4.8 \%$, and head: $1.1 \%$. Most individuals, $60.6 \%$, reported at least one secondary pain site.

Treatment was provided in a group format with the data of 16 consecutive groups included in the present analyses. Average group size was $6.9(S D=1.5)$ individuals. Previously published quantitative work has documented the effectiveness of the treatment program at the end of treatment and at a three month follow-up appointment (Vowles, Witkiewitz, Levell, Sowden, \& Ashworth, 2017; Vowles, Witkiewitz, Sowden, \& Ashworth, 2014). The material detailed in the present report has not previously been published. 


\section{Treatment details}

The interdisciplinary pain rehabilitation program included two days of treatment each week over a four-week period. Each treatment day included approximately 5.5 hours of active intervention provided by an interdisciplinary team. Specifically, each day included $1 \mathrm{~h}$ of physical conditioning training and exposure to avoided movements and $1 \mathrm{~h}$ of physical activity (e.g. mindful movement, Tai Chi, exposure to novel activities; activity planning). In addition, each day included 1.5 hours of psychological content, which included 30 minutes of mindfulness training, one hour of activity management (e.g., values clarification, goal setting), and one hour of educational or skills training content (e.g., pain physiology, effective communication, relapse preparation and prevention). The treatment team consisted of two physiotherapists, one clinical psychologist, one nurse, and one physician.

Treatment was based on the therapeutic model of Acceptance and Commitment Therapy (ACT), an intervention with "strong" empirical support, as graded by the American Psychological Association's Society of Clinical Psychology (Society of Clinical Psychology, 2011). The overall focus of ACT for chronic pain involves increasing adaptive responses to pain, particularly in patientidentified values, defined as domains of activity that bring meaning, importance, and vitality to the patient's life. To date, evidence suggests that ACT for chronic pain is associated with significant and sustained reductions in disability, improvements in overall emotional and physical functioning, greater engagement with valued activities, and fewer ineffective struggles for pain control (McCracken \& Vowles, 2014; Veehof, Trompetter, Bohlmeijer, \& Schreurs, 2016; Vowles \& Thompson, 2011).

\section{Data collection procedure}

Data was collected during the final treatment hour on the seventh day of the eight-day treatment program. The purpose of the session was to operationally define patient-identified adaptive behaviour changes that had occurred over the course of treatment. Colloquially, the treatment team referred to this session as the "survival guide" session in that its purpose was to derive a discrete and specific list of adaptive behaviour changes that would aid patients in "surviving" effectively with chronic pain once treatment had ended. 
The session was led by a clinical psychologist (KEV) and began by asking patients to identify actions taken since the beginning of treatment that had contributed to improvement or had been helpful in achieving improved values based action. The session leader then wrote down the actions identified by patients on a visual display (e.g., whiteboard, flipchart). A primary task of the session leader was to aid patients in defining discrete behavioural actions that had been taken, as opposed to outcomes that had been achieved by these actions. For example, if a patient noted that he or she was "doing better", then the patient was asked to clarify what had been done to occasion that improvement (e.g., "spend more time with family", "ask for help when I need it", "practice mindfulness"). Over the course of the 60 -minute session a list was generated by the group. The psychologist periodically asked patients whether the list was "complete", in the sense that engagement in the identified actions would aid in getting functioning back on track in relation to improved values in the event of a setback or that continued engagement in these actions would keep engagement in values at an adequate level for that patient. This question was used to aid in the generation of a more complete list and to aid in reinforcing a primary message in treatment, which was that treatment success involves effective responding to pain such that it does not needlessly limit one's quality of life. At the end of the session, all identified actions were typed out and distributed to patients so that the survival guide could be taken home by patients and used to help guide future behaviour.

\section{Transcription, overview of dataset and data analysis}

As described above, feedback from patient groups was transcribed by the session leader during the session. The comments generated by all the groups formed the data analysed in the present study. In total, the 16 treatment groups provided 315 unique comments. The number of comments collected from each group ranged in total from 13 to $35(M=19.7, S D=6.7)$. The number of characters in each comment ranged from 4 to $141(M=36.0, S D=20.6)$. The average comment was about as long as: "Worry less about what others think."

Given the nature of the dataset above, it was decided to analyse the comments using a mixed methods approach, i.e. both qualitative data analysis and simple, descriptive statistics. More specifically, thematic analysis (TA) was used to analyse the data. This method is regarded as a 
robust analytical technique that has flexibility in terms of approach, theory and epistemology (see Braun \& Clarke, 2006; Clarke \& Braun, 2013). Other, well-regarded qualitative methods were also considered, but ultimately not used as it was felt that these methods were better suited to different contexts. For example, Interpretative Phenomenological Analysis (IPA; Smith \& Osborn, 2008) tends to collect data in greater detail, from a smaller number of participants, often through semistructured interviews. In addition, a Grounded Theory approach was considered, but also not used as some argue that the end results need to involve the production of a "plausible / useful theory" (see Braun \& Clarke, 2006, p.81) - which this research did not set out to produce. Because this dataset consisted of hundreds of single, pre-existing comments, provided by individual patients within the context of a group treatment, it was felt that TA was the most appropriate method to use.

According to Braun and Clarke (2006), TA involves "searching across a data set... to find repeated patterns of meaning (p. 86)". Braun and Clarke describe six main phases to this process: transcribing the dataset, familiarizing oneself with the dataset; initial coding, searching for themes, reviewing and refining themes, and reporting the analysis. More specifically, in line with these guidelines, during the initial coding of the survival guide data, a representative specific theme was fitted to each comment, from each group. Each specific theme represented a short, subjective, descriptive label that aimed to summarise each comment. Generally speaking, only one code was allocated per comment. However, in certain instances, more than one code was needed, for example, when compound comments were analyzed.

As coding progressed, themes were identified, reviewed and refined. Refinement of the themes continued until the structure was perceived to adequately capture the observed data. Notes were used as a way of recording and exploring the process of allocating themes, helping to document the analytic process and achieve consensus across the two coders. The initial coding of the dataset was conducted by one coder (MT). The other coder (KEV) initially provided feedback and suggestions, and then independently rated comments to provide an index of inter-rater reliability with regard to the identified themes. The initial inter-rater reliability (Kappa) for all specific theme groups was 0.74 , indicating good initial agreement. The analysis resulted in a three level theme hierarchy: 1. Over-arching theme (the broadest category), 2. Mid-level theme, and 3. Sub- 
theme (the finest level of theme detail). In some areas, all three theme levels were needed to adequately capture the data, in others two levels sufficed.

Finally, in the spirit of reflexivity (see Braun \& Clarke, 2013, p.303), it is worth being explicit about the context, standpoints and perspective of the authors on this paper. As already noted, the interdisciplinary treatment programme the data was collected from follows an ACT approach. Moreover, many of the authors of this study have worked and published within the ACT domain for many years. Potential limitations of this perspective are more fully expanded in this discussion.

\section{Results}

For each of the overarching, mid-level, and sub-themes, representative quotes are provided to illustrate specific themes. In addition, simple, descriptive quantitative information is provided to order the themes in terms of decreasing prevalence across groups. We elected to provide more detail on themes that occurred in at least nine of the groups ( $>50 \%$ of groups). Themes that occur with less frequency are described in less detail.

In descending order of frequency, the three over-arching themes that emerged were: (1) Interacting with self (140 comments; $44 \%$ of total), (2) Activity (94 comments; 30\%), and (3) Interacting with others (69 comments, $22 \%)$. Of the total dataset, a small number of comments $(\mathrm{n}=$ 13) could not be clearly allocated as they fitted with multiple themes (4 comments; $1 \%$ ) or did not fit well within any of the identified overarching themes (9 comments, $3 \%)$. These 13 comments were not explored further.

\section{Overarching theme 1 : Interacting with self}

The 140 comments within this theme highlighted the interplay between various aspects of the individual. Each of the 16 groups had at least one comment related to this over-arching theme. Four mid-level themes are described below and shown in Figure 1.

$$
\text { [insert Figure } 1 \text { about here] }
$$

Mid-level theme 1.1: Relationship with private events. The most prevalent mid-leveltheme in this area was Relationship with private events (67 comments, 16 groups). The term "private events" refers to an individual's thoughts, feelings, memories, body sensations and urges and is derived from the radical behavioural approach that underlies ACT. It refers to behavioural 
events that are only observable to the individual who is behaving (Hayes \& Wilson, 1995; Moore, 2007; Skinner, 1953). As described elsewhere, ACT does not seek to alter the form or frequency that private events take, but instead seeks to alter the ways that these private events influence subsequent action. Every group made at least one comment related to this area. Figure 1 outlines the four sub-themes within this mid-level theme, as well as the frequency details.

The first sub-theme, labelled Flexibility/Willingness (34 comments, 15 groups), related to both flexibility in responding to, and willingness to experience, difficult private events. Some comments encapsulated this idea in a very succinct way: "Be flexible", "Struggle less". Other longer comments described it as: "Control the things you can control, leave the things you cannot control alone" and "See if you can be fully accepting and willing to be who you are right now". Some comments referred specifically to metaphors and experiential tasks used within the treatment program to help patients respond more flexibly and adaptively to private events. For example, some common ACT exercises ask patients to identify the ways that private events are unhelpfully influencing behaviour and to practice responding to them differently in order to maximize valued living. Reference to these exercises was made in several comments, such as: "Spot the passengers and notice them pushing you around" and "Don't let the passengers drive your life". This refers to the passengers on the bus exercise where "passengers" are thoughts and other private events on a metaphorical bus being driven through one's life (see Hayes, Strosahl, \& Wilson, 2011. p. 250).

The second sub-theme, Paying attention / Being aware (20 comments, 13 groups), included comments highlighting the importance of being able to monitor experience occurring in the present moment. As noted, each treatment day included 30 minutes of mindfulness exercises, which were designed specifically to augment present-focused awareness and effective responding to present experiences. The phrase "Be mindful" appeared in 7 of the 20 comments in this group. Other comments did not directly refer to mindfulness, but referred to other aspects of awareness and directed attention. For example: "Be aware of your choices" "and "Be in the here \& now, not the there \& then".

The third sub-theme was labelled, "Jump" / Take calculated risks (11 comments, 10 groups). 
This label highlights the notion of taking calculated risks to pursue values. "Jump" refers to an experiential exercise targeting willingness from the ACT tradition, related to the Zen saying: "You cannot jump a canyon in two steps" (see Hayes, Strosahl, \& Wilson, 1999, p. 240). The exercise suggests that while the "quantity" or height of any jump can be modified, the essential "quality" of jumping - i.e. putting yourself out into space and letting gravity do the rest (Hayes et al., 1999, p.241) remains vital and unchanged whatever the height. Comments ranged from the succinct: "Jump" to the longer "Take a chance when it matters (Jump!)". They also included other comments which appeared to capture the same sentiment without using the term jump: "Take risks in a controlled, sensible way". More specifically, of the 11 comments, 8 used the term "Jump", while another 3 mentioned taking risks. This sense of jumping / taking risks is another illustration of being flexible and willing (see earlier) in the pursuit of values-based activities, however the clustering of comments around these two terms seemed to warrant its own specific theme.

One additional sub theme was present, but was recorded in only two groups. It was labelled Responding to barriers (2 groups, 2 comments). One of these two comments, for example, was, "Stop using barriers as excuses."

Mid-level theme 1.2: Relationship with self. Relationship with self was the second most popular mid-level theme under the Interacting with self overarching theme (37 comments, $26 \%$ of main theme total; 16 groups). Again, every group made comments related to this area. The comments at this mid-level related to the individual's responses and reactions to him or herself. There were six sub-themes in this area, however, only one occurred in the majority of groups.

The largest sub-theme was Being gentle / Not beating one's self up (17 comments, 14 groups), and the content was closely related to the theme's title. For example: "Be gentle with yourself", "It is not necessary to beat yourself up" and "Don't beat yourself up when it doesn't achieve anything". In many ways, this sub-theme shares some similarities to earlier sub-themes that captured awareness of and flexibility with private events. However, in this particular subtheme, the focus was more on enhancing flexibility towards one's self more generally. As such it seemed to warrant its own specific theme.

The remaining five sub-themes only occurred in a minority of groups. Give credit to yourself 
(7 comments, 6 groups), Laugh (5 comments, 5 groups), Be honest with yourself (4 comments, 4 groups), and Trust / Believe in yourself (2 comments; 2 groups). Comments from these sub-themes closely mirrored the theme titles.

Mid-level theme 1.3: Values selection and awareness. The theme, labelled Values selection and awareness, was composed of 25 comments across 11 groups, representing $18 \%$ of the comments within the Interacting with self overarching theme. No sub-themes were necessary under this mid-level theme as all comments seemed to cohere sufficiently at the mid-level theme.

Within the ACT model, work around values highlights the things that are most important to an individual and seeks to ensure that they guide day to day behaviour (Hayes, Strosahl, et al., 2011). In ACT, a distinction is often made between values (a continuous direction of travel: e.g. to be a loving and caring partner) and goals (specific targets, big or small, in that direction of travel: e.g., to plan or attend an social event important to one's partner). In the patient comments, a number of comments referred to both values and goals, for example, "Keep values and goals in mind" and "Be guided by your values and goals". Other comments focused on these same things without specifically using the terms, for example values identification: "Have an aim", "Identify what you want", "Choose to do what you want" and values pursuit: "Do things that are worth it" and "Do more of what you want to do".

Mid-level theme 1.4: Relationship with pain. The fourth mid-level theme was labelled Relationship with pain, and consisted of comments describing an individual's interactions with the pain experience. Again, no further sub-themes were needed. Overall, there were 11 comments, across 9 groups, representing $8 \%$ of the total comments within the Interacting with self theme. Importantly, in keeping with the application of the ACT model in this area (Hayes, Villatte, Levin, \& Hildebrandt, 2011; McCracken \& Vowles, 2014; Vowles \& Thompson, 2011), all comments encapsulated the idea of either struggling less with pain (e.g., "Don't struggle needlessly with pain" and "Be wary of engaging in a struggle with pain. It saps energy that may be best put somewhere else") or engaging more with life, whilst also being willing to experience pain (e.g., "Live life with pain", "You will have pain regardless, so live your life").

\section{Overarching theme 2: Activity}


Activity was the second most prevalent overarching theme (94 comments, $30 \%$ of total comments), with relevant comments recorded across all 16 treatment groups. Whereas the preceding mid-level theme of values selection and awareness was related to being aware of what was most important to the individual, the comments in this overarching theme were more concerned with how individuals practically and sustainably move towards their identified values. The two mid-level themes: approach to activity and specific activity, and their sub-themes are described below.

\section{[insert Figure 2 about here]}

Mid-level theme 2.1: Approach to activities. The data in this mid-level theme (77 comments, 16 groups) relate to important qualities of overt behaviours and wider approaches to activity. The comments in this theme relate to these qualities and approaches rather than specific activities themselves. Each group provided a comment under this mid-level theme. Three subthemes were identified, all of which occurred in the majority of groups. Specifically: doing flexibly / pacing; dealing with setbacks / failure; making plans.

The largest sub-theme, labelled Doing flexibly / pacing (41 comments, 15 groups) was the most prevalent sub-theme in the entire data set. It refers to the spirit and, in some respects, speed at which activity is undertaken. On the one hand, comments repeatedly highlighted the idea of breaking down bigger behavioural objectives into sequential steps. In fact the word "steps" was mentioned in many comments: e.g. "Start with easy steps" and "Take things one step at a time". A similar sentiment was also expressed around pacing and slowing down: e.g. "Pace, don't be reckless in your behaviour" and "Be careful of rushing or pushing through". Equally, it was noted how it was "Ok to stop half way through" an activity, and also how it could be good to "Experiment" or, in slightly longer form: "Don't rule anything out and keep your options open". Ultimately, there was a sense in these comments that: "Quality matters more than quantity" and that doing just some of an activity slowly, but sustainably, was better in the long term than forcing oneself to hit a target in an unsustainable way.

The next sub-theme, Dealing with setbacks / failure (19 comments, 11 groups) made it clear that patients understood that setbacks could happen, were acceptable, and could even represent 
an opportunity to learn. For example, comments about the expected nature of set-backs included: "Expect setbacks", "It is ok to have a bad day" and "Fail (it is ok)". Equally, other comments focused on the potential to learn from setbacks: "Learn from your mistakes and learn from your experience", "Learn from your mistakes and try again", as well as the importance of re-engaging in behaviour: "After failing, make sure it matters and start again".

The final sub-theme, Make plans, (17 comments, 10 groups) encouraged individuals to make plans and set goals. The words: "plan", "goals" or "routine" regularly featured in specific comments, e.g., "Make and follow plans", "Set realistic goals", or "Establish a routine".

Mid-level theme 2.2: Specific activities. Comments within the Specific activities mid-level theme focused on the need to actively engage in specific activities. This focus on specific activity was in contrast to the more general approaches to activity captured in the previous mid-level theme. A total of 12 groups provided 17 comments under this mid-level theme. Three specific subthemes were identified, but none of these occurred in the majority of groups.

The first sub-theme, labelled Exercise (9 comments; 8 groups), placed priority on physical activity such as "Exercise", or "Get active/exercise/go to the gym". The second sub-theme, referred to more Generally doing activities (5 comments; 5 groups) and highlighted that one should concretely: "Put things into practice" and "Make a move towards what you want". The final subtheme, listed Specific other activities (3 comments, 3 groups) that an individual could do such as: "Get out of bed" or "Get out of the house".

\section{Overarching theme 3. Interacting with others}

The final overarching theme, Interacting with others, had the fewest comments of the overarching themes ( $n=69,22 \%$ of total comments). In total, 15 of 16 groups provided comments for this theme. The data in this theme shifted the focus from the relationship with one's own private events (overarching theme 1) to relationships with others. There were two mid-level themes, expanded below.

[insert Figure 3 about here]

Mid-level theme 3.1: Relationship with others. As shown in figure 3, The first mid-level theme, Relationship with others (36 comments, 15 groups) identified two sub-themes: Socializing 
and Focusing on self. The first, Socializing (19 comments, 15 groups), encouraged individuals to be around others and to avoid social isolation. For example: "Socialize/Don't isolate yourself", "Don't withdraw from others, socialize instead" and "Get around people who are like-minded". The second, Focusing on self (17 comments, 11 groups), encouraged people to do things for themselves. For example: "Take time for yourself", "Do something yourself" and "Do things by yourself". Also evident within this second specific theme was a focus on patients prioritizing themselves, for example by putting personal needs first. Examples included: "Take care of your own needs" and "Be selfish and put yourself first when it matters".

Mid-level theme 3.2: Communication with others. The final mid-level theme, labelled Communication with others (33 comments, 15 groups), reflected aspects of inter-personal communication. Four sub themes were identified.

The largest sub theme was labelled Asking for help, which was the only one observed in the majority of groups with 14 groups providing a total of 20 comments. Comments focused upon individuals asking for what was wanted or needed. Along with variations of: "Ask for help/Ask for what you want/Ask for what you need", other comments included: "Tell people what you want (they cannot read your mind)", "Talk to those who care about you and ask for help if you need it" and "Ask for and accept help when you need it".

The three remaining specific themes, each of which occurred in a minority of groups only included a focus on effectively Communicating feelings to others ( 6 comments; 6 groups), Listening to others (4 comments; 4 groups), and Saying no to requests when that was in one's best interest (3 comments; 3 groups).

\section{Discussion}

Over one hundred treatment completers, across 16 consecutive programmes, contributed data to a "survival guide" session. The results provide some initial insights into the patient perspective of salient adaptive behavioural changes gained as part of an interdisciplinary program of chronic pain rehabilitation. Three overarching themes were identified.

The first theme, "Interacting with self", included details of how patients had learned to interact with internal private events and aspects of their own behaviour. For example, comments indicated 
increased willingness to experience aversive private events, such as distress and pain; a broadening out of awareness beyond these experiences to become more aware of individual values, taking calculated risks in the direction of those values; as well as increases in selfcompassion. This theme included a number of behavioural interactions consistent with the ACT model of treatment, which itself prioritizes these ways of responding to aversive experiences if it promotes valued living (Hayes, Strosahl, et al., 2011; McCracken, 2005). Previous work using selfreport measures in chronic pain has also supported the importance of these response patterns in chronic pain, including acceptance of chronic pain (McCracken, 1998; McCracken, Vowles, \& Eccleston, 2004; Wicksell, Olsson, \& Hayes, 2010), more general willingness and acceptance (McCracken \& Zhao-O'Brien, 2010) and values clarity (McCracken \& Yang, 2006). Thus, when the patient comments under this theme are considered in light of what the model itself suggests are adaptive behaviours, a degree of concordance is apparent.

The second overarching theme ("Activity"), which pertained to patient engagement in activity, included changes in overall approach to activity, such as planning and flexible goal pursuit, adjusting to setbacks, as well as specific activities that were deemed useful to engage in. Overall, the activities within this theme were less ACT-specific and perhaps more in line with what we have previously referred to as "traditional" (McCracken \& Vowles, 2007; Vowles \& McCracken, 2010) or perhaps "more universal" pain specific coping behaviours. In other words, both ACT and non-ACT interdisciplinary pain rehabilitation place priority on improving strategic engagement in activity, facilitating judicious planning and pacing of activities, and decreasing sedentary or isolating behaviors (Fordyce, 1976; Gatchel, Peng, Peters, Fuchs, \& Turk, 2007; Jensen, Turner, Romano, \& Karoly, 1991; Main et al., 2015; Turk, Meichenbaum, \& Genest, 1983).

The final overarching theme described changes in social and interpersonal activity ("Interacting with others"). Patients noted the importance of improved social functioning, while also prioritizing their own needs. They also noted the importance of clarity in communication. As with the preceding theme, the facilitation of effective communication is often a part of interdisciplinary pain treatments, both ACT and non-ACT, thus its representation in patient comments is unsurprising.

Overall, these data appear to suggest that adaptive patient changes over the course of an 
interdisciplinary course of ACT for chronic pain was viewed by patients themselves as a mix of both ACT-specific and more universal coping / pain rehabilitation elements. This finding is broadly supported by some of our previous quantitative work which has indicated that both of these two "styles" of responding to pain increased over the course of a similar, but more intensive, ACT based interdisciplinary treatment (Vowles \& McCracken, 2010).

We conceive the separate themes presented above as being related to each other, and not completely distinct. This is perhaps illustrated by the values selection and awareness mid-level theme (of the interacting with self overarching theme) and the activity overarching theme. While it is acknowledged that the two exist in different theme hierarchies, it is also the case that what an individual does (activity) is hopefully influenced by what they personally value (values section and awareness). In this way, themes can be and are related, even when existing in different hierarchies.

[insert Table 1 about here]

Table 1 lists the most numerous themes in the data set. It is interesting that relationship with pain is the last entry in the table, and was only explicitly mentioned in 9 out of 16 groups. Although this may, at first, seem surprising, there are a number of ways of looking at this. Firstly, a lot of content concerning an individual's changing relationship with pain is included under other more common themes from Table 1 such as flexibility/willingness and paying attention / being aware. In other words, when participants in ACT interdisciplinary treatment increase flexibility with or awareness of private events, this includes thoughts and feelings, and it also explicitly includes body sensations such as pain. In addition, the focus of an ACT interdisciplinary treatment is not necessarily on reducing pain intensity or other symptoms, but on increasing daily functioning in the direction of values and goals even when pain reduction is not possible. Indeed, a recent study has even suggested that shifts in pain acceptance (not pain intensity or reduction) may be responsible for successful outcome in a CBT interdisciplinary treatment programme - even when pain acceptance was not explicitly targeted during treatment (see Akerblom, Perrin, Fischer, \& McCracken, 2015). Another recent study has indicated that improvements in pain intensity during treatment do not appear to be a pre-requisite for improvements in physical and emotional 
functioning following interdisciplinary ACT (Vowles, et al., 2017).

As qualitative methodology is not used with the same frequency as quantitative methodology in the chronic pain literature, it is worth placing this study in the context of other qualitative pain research. In their review of the qualitative literature, Osborn and Rodham (2010) note that previous chronic pain research has tended to focus on both the "lived experience of pain" and the personal experience of "seeking help for pain". The same review notes a possible discrepancy between qualitative and quantitative chronic pain research. Specifically, that the qualitative literature tends focus less on areas often found in the quantitative literature (i.e. "acceptance, fear avoidance and catastrophizing" [p.5]). It is noteworthy that the current research, using a qualitative methodology but focused on the end of treatment, primarily resulted in similar themes to those found in the quantitative literature. It seems possible that part of the reason for any discrepancy between qualitative and quantitative topic areas is simply down to the researcher area of interest. As this research was interested in patients adaptive behavioural insights gained as a result of interdisciplinary treatment, it seems natural that it reflects similar topics to those that are common in the mainly quantitative chronic pain treatment literature.

\section{Outcome and process research}

As highlighted briefly in the introduction, treatment research can be divided into that which focuses on outcome and that which focuses on process (see Kazdin, 2007). Outcome research concentrates primarily on whether a treatment works or how well it works in comparison to another treatment. Conversely process research, which is often found within psychotherapy and counselling areas, is more concerned with how a treatment works and what aspects of a treatment contribute to successful outcome. The work reported here examines treatment process, as it seeks to understand what aspects of treatment patients felt contributed to their behavioural changes. That said, the study also seeks to explore the possibility of linking future process findings to outcome data (see future research, below).

The process literature itself can arguably be divided into two camps: (1) that related to the importance of aspects of patient / provider relationship (also known as therapeutic alliance) and (2) that related to the importance of specific techniques within a given approach (see Castonguay, 
1993; Wampold, 2015). A number of researchers interested in process research argue for the primacy of the patient / provider relationship (see Norcross \& Lambert, 2011; Norcross \& Wampold, 2011). Regular, peer-reviewed, meta-analyses have examined the influence of this relationship on psychotherapeutic outcome and have found a remarkably consistent but relatively small correlation (between 0.22 and 0.28 ), accounting for between about $5 \%$ to $8 \%$ of the variance (see Vowles \& Thompson, 2012). It is noteworthy that such research tends to focus on the working together of an individual therapist and an individual client. Of course, in the chronic pain treatment field, treatment often involves larger groups of patients, who regularly see several different health care professionals concurrently as part of their interdisciplinary treatment.

With this in mind, it is worth noting two things. Firstly, that the influence of the various interand intra- patient / provider relationships in group based pain management programmes might be both significant and complex (see, for example, Lewis et al., 2010; Williams \& Potts, 2010). Secondly, as this specific research only drew upon material from the survival guide session (see methods), such data is only likely to pick up on material related to specific techniques within an approach. It is not likely to highlight material related to aspects of the patient / provider relationship. This of course, does not mean that such relational material was not important, only that this data was not likely to be collected as part of the survival guide session. In some ways, this must be considered a limitation of this research. Further limitations are highlighted below.

\section{Limitations}

As with much research of this nature, the final overarching, mid-level and sub-themes were reached through analysis and discussions between a limited number of researchers. Despite reflective checks, it is possible that another team may have categorised the data differently. As noted above, it is important to note that the dividing lines between the final themes is likely in some way to be a reflection of the researchers and their interests, and a different set of researchers may have found other plausible arrangements of the data (see future research below).

The data that makes up this dataset comes from inside a "real life" chronic pain treatment environment. Equally, similar to previous qualitative research of this type (e.g. Dunford, Thompson, \& Gauntlett-Gilbert, 2014), this data does not come from isolated individuals, but from individuals 
who were part of a treatment group as a whole. As noted in the method, survival guide comments were suggested by patients in the group and discussed as a group, as they were being written up on the white board by the session leader. Naturally there is a process of filtering and refinement that takes place here, and perhaps some level of influence from both the wider group and the session leader. This may limit the extent to which these comments can be seen to reflect the thoughts of any specific individual within any one group. However, the above notwithstanding, the data still seems to capture potentially interesting insights.

\section{Future research and conclusion}

In their review, Osborn and Rodham (2010) noted that researchers tended not to "accumulate a substantial body of [qualitative] work" enabling them "to develop their arguments" (p. 5) over time. With this is mind it is worth considering what future research in this area may look like. It would be interesting if other interdisciplinary treatment programmes were to see the "survival guide" session as a potentially useful clinical tool. If adopted, this could allow for future data collection and analysis that replicates the above work. It would be interesting to see if similar themes emerged from replicated research conducted within treatment programmes from different therapeutic orientations, for example, traditional Cognitive Behavioural Therapy (CBT). It seems quite possible that many of the traditional coping behaviours from themes 2 and 3 would be similar given the shared lineage of both ACT and CBT - although, of course, further research would be required to evidence this.

In future work, it will be important to examine the extent to which the themes identified in this work fit with new data or whether revisions are needed. For example, one thing that may happen over time is that some themes that did not feature in half or more of the groups, may become more (or less) prominent. Moving even more towards a mixed methods approach, it also seems possible that with enough data, future research could examine whether the differences in the existence and the extent of qualitative themes has any relation to the improvements in physical and emotional functioning that are associated with the treatment itself. Thus, more directly attempting to use this qualitative data to examine the patient reported processes that influence quantitative outcome.

In conclusion, this paper provides a preliminary, mainly qualitative, exploration into the 
adaptive behavioural insights gained by patients during an interdisciplinary programme of chronic pain rehabilitation. Importantly, the paper provides contemporaneous qualitative evidence of the active ingredients of therapeutic change from the patient point of view; an area that thus far appears to have been underserved by the chronic pain literature. The specific findings of this research highlight the perceived importance of both ACT-specific and more universal coping / pain rehabilitation elements within an ACT focused interdisciplinary treatment programme. The connections between the findings in this paper and findings previously reported in the quantitative assessment and treatment literature may go some way to both help explain and even potentially bridge a gap that has existed between the qualitative and quantitative chronic pain literatures. Importantly, the methodology adopted here may also be adopted by other services, using other frameworks to help continue to pursue process research in this area. Future research following these methods may further investigate the generalisability of these findings and how they translate across programmes of different orientations. Finally, extensions of this work may even have the potential to examine more closely how these potentially relevant processes relate more directly to treatment outcome: marrying the traditions of process and outcome research. 


\section{References}

Akerblom, S., Perrin, S., Fischer, M. R., \& McCracken, L. M. (2015). The mediating role of acceptance in multidisciplinary cognitive-behavioral therapy for chronic pain. J Pain, 16(7), 606615.

Bergström, G., Bergström, C., Hagberg, J., Bodin, L., \& Jensen, I. (2010). A 7-year follow-up of multidisciplinary rehabilitation among chronic neck and back pain patients. Is sick leave outcome dependent on psychologically derived patient groups? Eur J Pain, 14(4), 426-433.

Braun, V., \& Clarke, V. (2006). Using thematic analysis in psychology. Qual Res Psychol, 3(2), $77-101$.

Braun, V., \& Clarke, V. (2013). Successful qualitative research: A practical guide for beginners. London: Sage Publications.

British Pain Society. (2013). Guidelines for pain management programmes for adults: An evidence-based review prepared on behalf of the British Pain Society. London: British Pain Society.

Castonguay, L. G. (1993). "Common factors" and "nonspecific variables": Clarification of the two concepts and recommendations for research. J Psychother Integr, 3(3), 267-286.

Clarke, V., \& Braun, V. (2013). Teaching thematic analysis: Over- coming challenges and developing strategies for effective learning. Psychologist, 26(2), 120-123.

Dunford, E., Thompson, M., \& Gauntlett-Gilbert, J. (2014). Parental behaviour in paediatric chronic pain: A qualitative observational study. Clin Child Psychol Psychiatry, 19(4), 561-575.

Flor, H., Fydrich, T., \& Turk, D. C. (1992). Efficacy of multidisciplinary pain treatment centers: a meta-analytic review. Pain, 49(2), 221-230.

Fordyce, W. E. (1976). Behavioral Methods for Chronic Pain and Illness. (St. Louis: CV Mosby).

Gatchel, R. J., McGeary, D. D., McGeary, C. A., \& Lippe, B. (2014). Interdisciplinary chronic pain management: past, present, and future. Am Psychol, 69(2), 119-130.

Gatchel, R. J., \& Okifuji, A. (2006). Evidence-based scientific data documenting the treatment and cost-effectiveness of comprehensive pain programs for chronic nonmalignant pain. J Pain, 7(11), 779-793. 
Gatchel, R. J., Peng, Y. B., Peters, M. L., Fuchs, P. N., \& Turk, D. C. (2007). The biopsychosocial approach to chronic pain: Scientific advances and future directions. Psychol Bull, 133(4), 581-624.

Hatten, A. L., Gatchel, R. J., Polatin, P. B., \& Stowell, A. W. (2006). A cost-utility analysis of chronic spinal pain treatment outcomes: converting SF-36 data into quality-adjusted life years. The Clin J Pain, 22(8), 700-711.

Hayes, S. C., Strosahl, K. D., \& Wilson, K. G. (1999). Acceptance and commitment therapy: An experiential approach to behavior change. New York, New York: Guilford Press.

Hayes, S. C., Strosahl, K. D., \& Wilson, K. G. (2011). Acceptance and commitment therapy: The process and practice of mindful change. New York, New York: Guilford Press.

Hayes, S. C., Villatte, M., Levin, M., \& Hildebrandt, M. (2011). Open, aware, and active: Contextual approaches as an emerging trend in the behavioral and cognitive therapies. Annu Rev Clin Psychol, 7(1), 141-168.

Hayes, S. C., \& Wilson, K. G. (1995). The role of cognition in complex human behavior: A contextualistic perspective. J Behav Ther Exp Psychiatry, 26(3), 241-248.

Jensen, I. B., Bergström, G., Ljungquist, T., \& Bodin, L. (2005). A 3-year follow-up of a multidisciplinary rehabilitation programme for back and neck pain. Pain, 115(3), 273-283.

Jensen, M. P., Turner, J. A., Romano, J. M., \& Karoly, P. (1991). Coping with chronic pain: a critical review of the literature: Pain, 47(3), 249-283.

Kamper, S. J., Apeldoorn, A. T., Chiarotto, A., Smeets, R. J., Ostelo, R. W., Guzman, J., \& van Tulder, M. W. (2014). Multidisciplinary biopsychosocial rehabilitation for chronic low back pain. The Cochrane Library. Retrieved from http://onlinelibrary.wiley.com/doi/10.1002/14651858.CD000963.pub3/pdf

Kamper, S. J., Apeldoorn, A. T., Chiarotto, A., Smeets, R. J., Ostelo, R. W., Guzman, J., \& van Tulder, M. W. (2015). Multidisciplinary biopsychosocial rehabilitation for chronic low back pain: Cochrane systematic review and meta-analysis. BMJ, 350, h444.

Kazdin, A. E. (2007). Mediators and Mechanisms of Change in Psychotherapy Research. Annu. Rev. Clin. Psychol, 3(1), 1-27. 
Lewis, M., Morley, S., van der Windt, D. A. W. M., Hay, E., Jellema, P., Dziedzic, K., \& Main, C. J. (2010). Measuring practitioner/therapist effects in randomised trials of low back pain and neck pain interventions in primary care settings. Eur J Pain, 14(10), 1033-1039.

Main, C. J., Keefe, F. J., Jensen, M. P., Vlaeyen, J. W., \& Vowles, K. E. (2015). Fordyce's Behavioral Methods for Chronic Pain and Illness: Republished with invited commentaries. (New York: Walters-Kluwer).

McCracken, L. M. (1998). Learning to live with the pain: acceptance of pain predicts adjustment in persons with chronic pain. Pain, 74(1), 21-27.

McCracken, L. M. (2005). Contextual Cognitive-behavioral Therapy for Chronic Pain. (Seattle: ISAP Press).

McCracken, L. M., \& Vowles, K. E. (2007). Psychological flexibility and traditional pain management strategies in relation to patient functioning with chronic pain: an examination of a revised instrument. J Pain, 8(9), 700-707.

McCracken, L. M., \& Vowles, K. E. (2014). Acceptance and commitment therapy and mindfulness for chronic pain: Model, process, and progress. Am Psychol, 69(2), 178-187.

McCracken, L. M., Vowles, K. E., \& Eccleston, C. (2004). Acceptance of chronic pain: component analysis and a revised assessment method. Pain, 107(1-2), 159-166.

McCracken, L. M., \& Yang, S. Y. (2006). The role of values in a contextual cognitive-behavioral approach to chronic pain. Pain, 123(1-2), 137-145.

McCracken, L. M., \& Zhao-O’Brien, J. (2010). General psychological acceptance and chronic pain: There is more to accept than the pain itself. Eur J Pain, 14(2), 170-175.

Moore, J. (2007). Conceptual Foundations of Radical Behaviorism. NY: Sloan Publishing.

Norcross, J. C., \& Lambert, M. J. (2011). Psychotherapy Relationships That Work II. Psychotherapy, 48(1), 4-8.

Norcross, J. C., \& Wampold, B. E. (2011). Evidence-Based Therapy Relationships: Research Conclusions and Clinical Practices. Psychotherapy, 48(1), 98-102.

O’Brien, E. M., Staud, R. M., Hassinger, A. D., McCulloch, R. C., Craggs, J. G., et al. (2010). Patient-centered perspective on treatment outcomes in chronic pain. Pain Med, 11(1), 6-15. 
Osborn, M., \& Rodham, K. (2010). Insights into pain: a review of qualitative research. Rev Pain, 4(1), 2-7.

Skinner, B. F. (1953). Science and Human Behavior. New York, New York: Simon and Schuster

Smith, J. A., \& Osborn, M. (2008). Interpretative Phenomenological Analysis. In Qualitative Psychology: A Practical Guide to Research Methods (pp. 53-80). London: Sage Publications.

Society of Clinical Psychology. (2011). Acceptance and Commitment Therapy for Chronic Pain. Retrieved March 2, 2017, from https://www.div12.org/psychological-treatments/disorders/chronicor-persistent-pain/acceptance-and-commitment-therapy-for-chronic-pain/

Thorne, F. M., \& Morley, S. (2009). Prospective judgments of acceptable outcomes for pain, interference and activity: patient-determined outcome criteria. Pain, 144(3), 262-269.

Turk, D. C. (2002). Clinical effectiveness and cost-effectiveness of treatments for patients with chronic pain. Clin J Pain, 18(6), 355-365.

Turk, D. C., \& Burwinkle, T. M. (2005). Clinical outcomes, cost-effectiveness, and the role of psychology in treatments for chronic pain sufferers. Prof Psychol Res Pr, 36(6), 602-610.

Turk, D. C., Meichenbaum, D., \& Genest, M. (1983). Pain and Behavioral Medicine: A Cognitive-behavioral perspective. (New York: Guildford Press).

Veehof, M. M., Trompetter, H. R., Bohlmeijer, E. T., \& Schreurs, K. M. G. (2016). Acceptanceand mindfulness-based interventions for the treatment of chronic pain: a meta-analytic review. Cogn Behav Ther, 45(1), 5-31.

Vowles, K. E., \& McCracken, L. M. (2010). Comparing the role of psychological flexibility and traditional pain management coping strategies in chronic pain treatment outcomes. Behav Res Ther, 48(2), 141-6.

Vowles, K. E., McCracken, L. M., \& O’Brien, J. Z. (2011). Acceptance and values-based action in chronic pain: a three-year follow-up analysis of treatment effectiveness and process. Behav Res Ther, 49(11), 748-755.

Vowles, K. E., \& Thompson, M. (2011). Acceptance and commitment therapy for chronic pain. In Mindfulness and Acceptance in Behavioral Medicine: Current Theory and Practice, L. McCracken ed. (Oakland, CA: New Harbinger). pp. 31-60. 
Vowles, K. E., \& Thompson, M. (2012). The Patient-Provider Relationship in Chronic Pain. Curr Pain Headache Rep, 16(2), 133-138.

Vowles, K. E., Witkiewitz, K., Levell, J., Sowden, G., \& Ashworth, J. (2017). Are reductions in pain intensity and pain-related distress necessary? An analysis of within-treatment change trajectories in relation to improved functioning following interdisciplinary acceptance and commitment therapy for adults with chronic pain. Journal of Consulting and Clinical Psychology, 85(2), 87-98.

Vowles, K. E., Witkiewitz, K., Sowden, G., \& Ashworth, J. (2014). Acceptance and Commitment Therapy for Chronic Pain: Evidence of Mediation and Clinically Significant Change Following an Abbreviated Interdisciplinary Program of Rehabilitation. J Pain, 15(1), 101-113.

Wampold, B. E. (2015). How important are the common factors in psychotherapy? An update. World Psychiatry, 14(3), 270-277.

Wicksell, R. K., Olsson, G. L., \& Hayes, S. C. (2010). Psychological flexibility as a mediator of improvement in Acceptance and Commitment Therapy for patients with chronic pain following whiplash. European Journal of Pain, 14(10), 1059.e1-1059.e11.

Williams, A. C. de C., Eccleston, C., \& Morley, S. (2012). Psychological therapies for the management of chronic pain (excluding headache) in adults. The Cochrane Library. Retrieved from http://onlinelibrary.wiley.com/doi/10.1002/14651858.CD007407.pub3/pdf

Williams, A. C. de C., \& Potts, H. W. W. (2010). Group membership and staff turnover affect outcomes in group CBT for persistent pain. Pain, 148(3), 481-486. 
Figures legend

Figure 1: Overarching, mid-level and sub themes in the "Interacting with self" theme hierarchy.

Figure 2: Overarching, mid-level and sub themes in the "Activity" theme hierarchy.

Figure 3: Overarching, mid-level and sub themes in the "Interacting with others" theme hierarchy. 


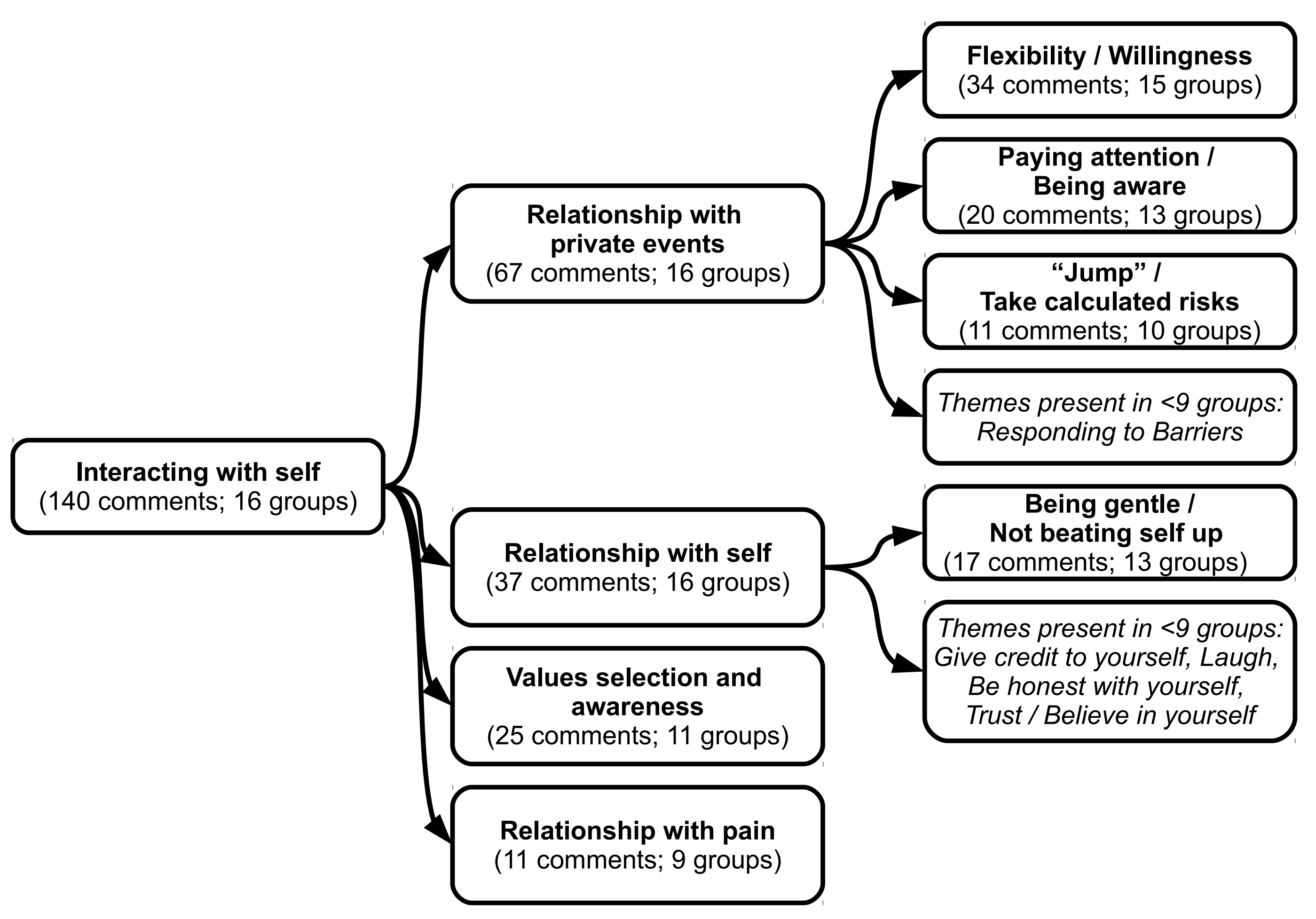




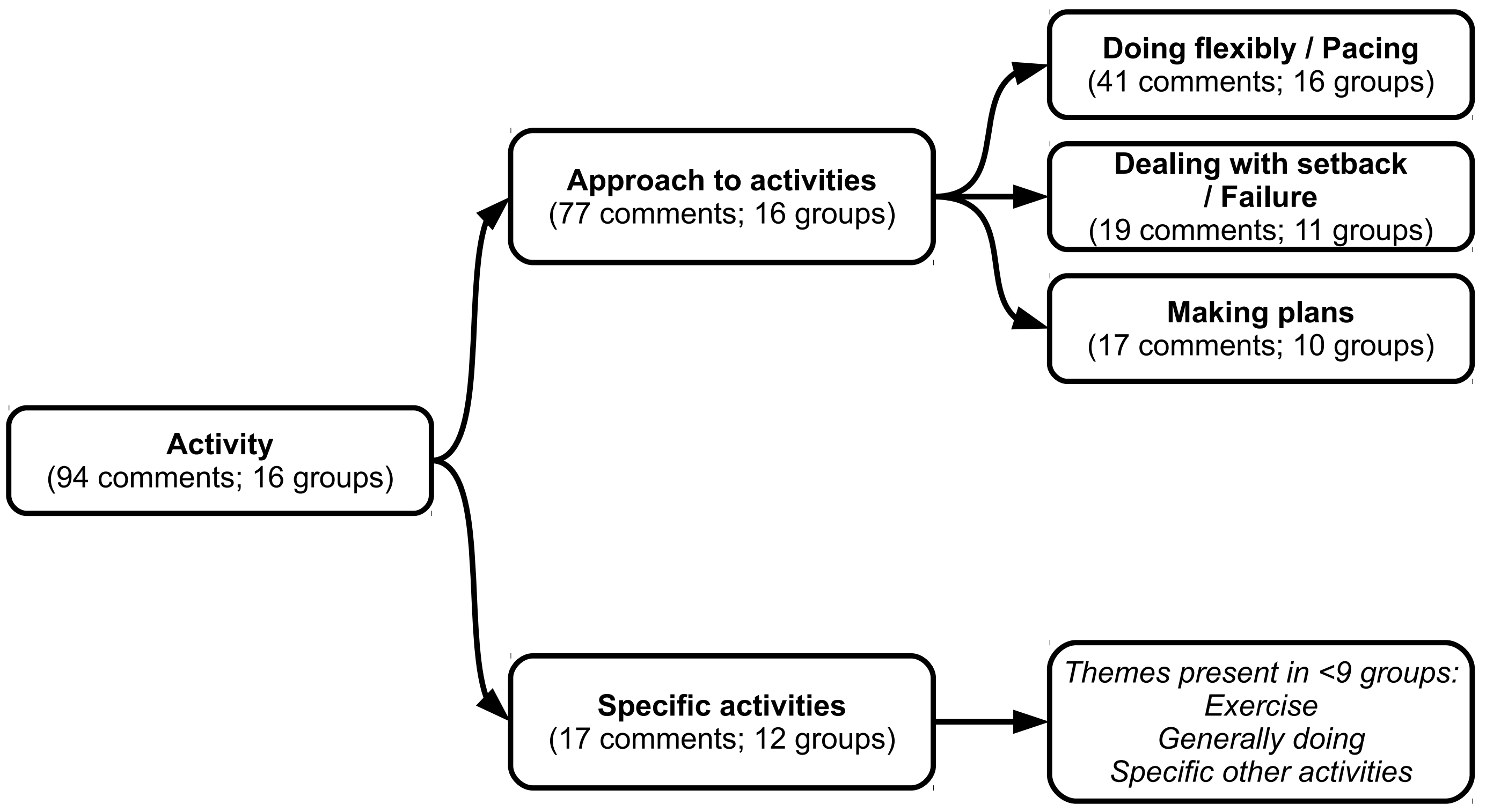




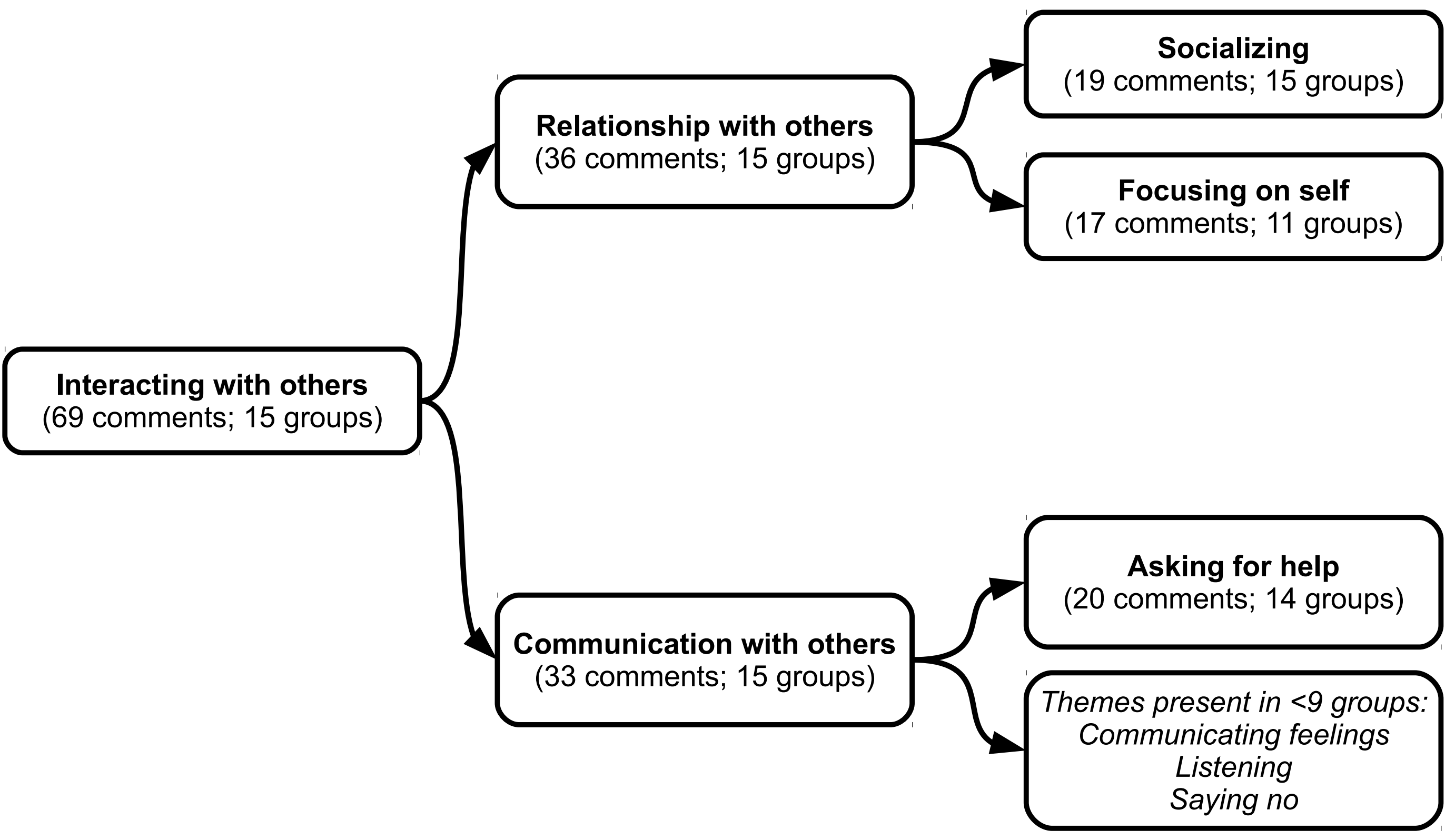

